\title{
Vestibular Syndromes in Childhood and Adolescence
}

\author{
Juliana Antoniolli Duarte ${ }^{1}$ Elisa Morais Leão ${ }^{1}$ Daniel Sobral Fragano ${ }^{1}$ Germana Jardim Marquez ${ }^{1}$ \\ Anna Paula Batista de Ávila Pires ${ }^{1}$ Maria Laura Solferini Silva ${ }^{1}$ Fernando Freitas Ganança ${ }^{1}$ \\ ${ }^{1}$ Department of Otorhinolaryngology and Head and Neck Surgery, \\ Escola Paulista de Medicina, Universidade Federal de São Paulo \\ (Unifesp), São Paulo, SP, Brazil \\ Int Arch Otorhinolaryngol 2020;24(4):e477-e481. \\ Address for correspondence Juliana Antoniolli Duarte, MSc, \\ Departmento de Otorrinolaringologia e Cirurgia de Cabeça e Pescoço, \\ Escola Paulista de Medicina, Universidade Federal de São Paulo \\ (Unifesp), Rua Pedro de Toledo, 947, Vila Clementino, São Paulo, \\ SP, 04039-002, Brazil (e-mail: j_antoniolli@yahoo.com.br).
}

\begin{abstract}
Introduction The prevalence of vestibular disorders in childhood ranges from $0.4 \%$ to $15 \%$; they may be the result of several factors, but most of the time it's an episodic vestibular syndrome related to migraine equivalents.

Objective To evaluate the diagnostic and therapeutic aspects of children with vestibular signs and symptoms.

Methods The present cross-sectional study evaluated data from the records of patients treated in an outpatient pediatric neurotology clinic over a 10-year period. These data included sociodemographic and clinical variables, results of complementary examinations, the treatment provided, and the clinical evolution.

Results The sample was composed of 117 patients, with $54.7 \%$ of female subjects with a mean age of 10 years. The most prevalent diagnosis was benign paroxysmal vertigo of childhood (BPVC) (41.9\%), followed by vestibular migraine (16.2\%). The most prevalent complaint was vertigo (53.9\% of the cases). Most patients $(66.7 \%)$ had inadequate eating habits. Improvement of symptoms was observed in $40.4 \%$ of the patients treated with dietary guidance alone. In $80 \%$ of the cases, dietary counseling in

Keywords

- child

- vertigo

- migraine disorders

- dizziness combination with vestibular rehabilitation therapy achieved therapeutic success without the need of a drug treatment.

Conclusion The predominant diagnosis was of BPVC, and its close relationship with the personal and family history of migraine, its benign evolution, and the importance of dietary guidance and vestibular rehabilitation for therapeutic success were observed.
\end{abstract}

\section{Introduction}

Balance in the body is comprised of the integration of the visual, vestibular, and somatosensory systems, which is regulated by the central nervous system (CNS) through cerebellum activity. These systems are anatomically developed at birth, but seamless integration between them only occurs at around 8 to 10 years of age. Any conflict between these sensory systems can cause vestibular symptoms (VSs). ${ }^{1}$

The prevalence of vestibular disorders in childhood ranges from $0.4 \%$ to $15 \%,{ }^{2,3}$ and may be underestimated as a result of several factors: vestibular alterations are usually ignored in children; expressions of disrupted bodily balance are usually attributed to lack of motor coordination or behavioral changes; ${ }^{4}$ children have difficulty expressing their symptoms; ${ }^{5}$ and the most prevalent causes of VS differ in adults and children. ${ }^{6}$

The most prevalent causes of VS in children and adolescents are benign paroxysmal vertigo of childhood (BPVC), serous otitis media (SOM), vestibular migraine (VM), head trauma, malformation of the inner ear, vestibular neuritis, infectious labyrinthitis, visual changes, and tumors of the posterior fossa. ${ }^{2-13}$

Conventionally, in addition to the clinical examination, vestibular investigation in children includes examinations such as rotary chair testing, caloric testing, and posturography. Other tests to evaluate vestibular function, such as the videohead impulse test (VHIT) and vestibular-evoked myogenic received

August 9, 2019

accepted

November 3, 2019
DOI https://doi.org/

10.1055/s-0039-3402443. ISSN $1809-9777$.
Copyright $(2020$ by Thieme Revinter

Publicações Ltda, Rio de Janeiro, Brazil
License terms

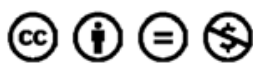


potentials (VEMPs), have recently been introduced for use in the pediatric population. ${ }^{1,14}$

Considering vestibular disorders in childhood and adolescence is essential so they can be recognized early; delays in treatment can affect other aspects of development such as spoken and written language, psychological state, and school performance. $^{2,4}$ Consequently, the objective of the present study was to analyze the profile of children and adolescents with VS who were cared for in an outpatient pediatric neurotology clinic, including the diagnostic and therapeutic aspects.

\section{Materials and Methods}

The present cross-sectional observational study evaluated all records of patients up to 18 years of age treated in an outpatient pediatric neurotology clinic from 2010 to 2019. Illegible records were excluded. We evaluated the following descriptive factors: gender; age range; clinical diagnosis and associated diagnoses; eating habits; VSs; auditory symptoms; personal history of headache and motion sickness; family history of migraine; time of disease progression; laboratory examinations; caloric tests (performed in children aged 5 years and older); audiometry and immittance audiometry; imaging tests, such as computed tomography (CT) of the skull/temporal bones and magnetic resonance imaging (MRI) of the brain/inner ears; the treatments performed; and the clinical progress.

Subsequently, the patients were divided into three groups according to the clinical diagnosis for analytic assessment: group 1 - patients diagnosed with BPVC; group 2 - patients diagnosed with VM; and group 3 - patients with other diagnoses (not BPVC or VM). Associations were made between these groups for three variables: presence or absence of headache, family history of migraine, and motion sickness.

The data were stored and analyzed in an Excel (Microsoft Corp., Redmond, WA, US) spreadsheet, and the associations among the data were evaluated using a homogeneous onetailed $t$-test at a $95 \%$ significance level; when differences were found among the three groups, the heterogeneous onetailed $t$-test was used.

\section{Results}

We assessed a total of 144 patient records, and 117 were considered eligible for inclusion. The included patients were between 2 and 17 years of age, with a mean age of 10 years (standard deviation [SD]: 3.62). Of these, 64 (54.70\%) were female.

The most prevalent diagnosis was of BPVC, which was found in 49 cases (41.9\%); VM was found in 19 patients (16.2\%); motion sickness, in 11 (9.50\%); metabolic vestibular syndrome (MVS), in 7 (6\%); CNS changes (epilepsy, sequelae of meningoencephalitis, and Chiari malformation), in 3 patients (2.50\%); Ménière disease in 3 cases (2.50\%); dysautonomia in 3 patients (2.50\%); benign paroxysmal positional vertigo (BPPV) in 2 cases (1.7\%); and labyrinthine concussion in 2 cases (1.70\%). Other less prevalent diagnoses were: 1 case of SOM $(0.8 \%) ; 1$ case of superior semicircular canal dehiscence $(0.8 \%) ; 1$ case of autoimmune endolymphatic hydrops $(0.8 \%)$; and, in 15 cases $(12.8 \%)$, a conclusive diagnosis could not be established.

Poor eating habits were reported by 78 patients $(66.7 \%)$, with high carbohydrate intake predominating in 68 cases (57.95\%), followed by prolonged fasting in 21 cases (23.86\%). Excluding patients who did not report their eating habits, the percentage of patients with poor eating habits increased to $75,50 \%$ (78); of these, $82,10 \%$ (64) overconsumed carbohydrates (sweets, chocolate, pasta, bread, and soft drinks) and $13,20 \%$ (10) habitually fasted for long periods of time.

Positive family history was present in 84 patients (71.79\%), predominantly history of migraine 53 (45,29\%), followed by diabetes mellitus 25 (21,66\%).

As for the clinical condition, most participants 64 (54,54\%) complained of vertigo while 29 (24.78\%) patients complained of dizziness; of these, 6 cases (5.12\%) were cases of nausea-type dizziness, while only 5 (4.27\%) patients complained of imbalance. Instability was only described in 2 ( $1.70 \%)$ cases, gait deviation, in $1(0.85 \%)$ case, and $3(2.56 \%)$ patients reported a falling sensation.

Of the patients with hearing complaints (47), 20 (42,55\%) of the cases presented hearing loss as an isolated complaint, and 13 (27,65\%) complained of tinnitus. Audiometry was performed in 84 patients, and alterations were only found in 24 cases $(20.51 \%) ; 16(66,7 \%)$ of these cases showed neurosensory loss, and $8(33,3 \%)$ of patients showed conductivetype loss. Immittance audiometry only showed alterations in $1(1,2 \%)$ case, with a B curve and a diagnosis of SOM.

Electronystagmography (ENG) was performed on 60 patients $(51.28 \%)$, yielding results within normal limits in $23.1 \%$ (27) of these cases. There were no changes in the oculomotor examinations; the caloric tests showed that 18 (30\%) patients had bilateral hyperreflexia, 8 (13.33\%) patients had unilateral hyperreflexia, $2(3.33 \%)$ presented unilateral deficit, and 1 (1.66\%) patient presented bilateral deficit. Among the 49 cases of BPVC, caloric testing yielded normal results in 9 cases (18,3\%), bilateral hyperreflexia was found in 10 patients $(20,4 \%)$, and unilateral hyperreflexia, in 5 patients (10,2\%). Only 1 patient (2\%) exhibited a bilateral deficit, and 2 patients (4\%) presented unilateral deficit.

The use of VHIT and VEMPs is still recent in the pediatric age group, and only two children were examined: one with Ménière disease and the other with VM. The results were normal in both cases.

A brain MRI was requested for 13 (12.5\%) patients, and alterations were only observed in 2 cases $(15,4 \%)$, (Chiari malformation and expansive cystic lesion in the temporal fossa), and an MRI of the inner ear was requested in 1 case (7,7\%) and was normal. A skull CT was requested for 3 patients (2,56\%) and showed no alterations; a CT of the temporal bones was requested in 3 cases, and in 1 case $(33,3 \%)$ it showed a malformation of the inner ear in the anterior labyrinth.

Clinical improvement was observed in 60 (51.28\%) patients over an average period of 27 months.

In total, 50 patients $(42,73 \%)$ received dietary guidance alone as a treatment, and, of this subgroup, 26 (52\%) improved. Among the patients who received dietary guidance and/or a 
Table 1 Prevalence of headache among patients diagnosed with benign paroxysmal vertigo of childhood and vestibular migraine and patients with other diagnoses

\begin{tabular}{|l|l|l|l|l|l|l|l|}
\hline & \multicolumn{2}{|l|}{ Yes } & No & & \multicolumn{2}{l|}{ Total } & $p$-value \\
\hline Group 1 & 41 & $85.4 \%$ & 7 & $14.6 \%$ & 48 & $100 \%$ & \\
\hline Group 2 & 18 & $94.7 \%$ & 1 & $5.3 \%$ & 19 & $100 \%$ & 0.0035 \\
\hline Group 3 & 30 & $65.2 \%$ & 16 & $34.8 \%$ & 46 & $100 \%$ & \\
\hline Total & 89 & $78.8 \%$ & 24 & $21.2 \%$ & 113 & $100 \%$ & \\
\hline
\end{tabular}

Notes: Group 1 - patients diagnosed with benign paroxysmal vertigo of childhood; group 2 - patients with diagnosis of vestibular migraine; group 3 - patients with other diagnoses.

combination of dietary guidance and vestibular rehabilitation, $60 \%$ (26) showed improvement. A combination of diet and medications was used to treat 23 patients (19,5\%), and treatment was successful in $69,56 \%$ (16) of this group.

The combination of diet and prescription medications produced success in 21 (17.94\%) cases; 1 patient underwent tympanotomy to place a ventilation tube, and the Epley maneuver was performed in 2 patients. One patient was referred for auditory prosthesis, 3 patients were referred for neurology assessment and monitoring, 1 case was referred for psychological evaluation, 1 case, for genetic evaluation, and 1 patient, for orthopedic evaluation.

Comparisons between the 3 groups of patients according to the etiological diagnosis for the prevalence of the variables of complaints of headache, presence or absence of family history of migraine, and presence or absence of motion sickness can be seen in -Tables 1-3 respectively.

Note that in -Tables $\mathbf{1}$ and $\mathbf{2}$ there was a statistically significant difference among the groups regarding the presence of headache complaints and family history of migraine, which did not occur with respect to the presence of motion sickness. For the first two variables, we only found a difference between groups 1 and 3 , of 0.007 and 0.017 respectively.

\section{Discussion}

The average age described for the diagnosis of vestibular disorders in children ranges from 7 to 11 years; ${ }^{6}$ diagnosis is most frequent around age $10 .^{2,5,15}$ In the present study, we observed an average age of 10 years. A different prevalence

Table 2 Prevalence of family history of migraine among patients diagnosed with benign paroxysmal vertigo of childhood and vestibular migraine and patients with other diagnoses

\begin{tabular}{|l|l|l|l|l|l|l|l|}
\hline & \multicolumn{2}{|l|}{ Yes } & No & & \multicolumn{2}{l|}{ Total } & $p$-value \\
\hline Group 1 & 37 & $75.5 \%$ & 12 & $24.5 \%$ & 49 & $100 \%$ & \\
\hline Group 2 & 6 & $54.5 \%$ & 5 & $45.5 \%$ & 11 & $100 \%$ & 0.04042 \\
\hline Group 3 & 18 & $54.5 \%$ & 15 & $45.5 \%$ & 33 & $100 \%$ & \\
\hline Total & 61 & $65.6 \%$ & 32 & $34.4 \%$ & 93 & $100 \%$ & \\
\hline
\end{tabular}

Notes: Group 1 - patients diagnosed with benign paroxysmal vertigo of childhood; group 2 - patients with diagnosis of vestibular migraine; group 3 - patients with other diagnoses.
Table 3 Prevalence of motion sickness in patients diagnosed with benign paroxysmal vertigo of childhood and vestibular migraine and patients with other diagnoses

\begin{tabular}{|l|l|l|l|l|l|l|l|}
\hline & \multicolumn{2}{|l|}{ Yes } & No & & \multicolumn{2}{l|}{ Total } & $p$-value \\
\hline Group 1 & 34 & $69.4 \%$ & 15 & $30.6 \%$ & 49 & $100 \%$ & \\
\hline Group 2 & 7 & $38.9 \%$ & 11 & $61.1 \%$ & 18 & $100 \%$ & 0.26845 \\
\hline Group 3 & 25 & $54.3 \%$ & 21 & $45.7 \%$ & 46 & $100 \%$ & \\
\hline Total & 66 & $58.4 \%$ & 47 & $41.6 \%$ & 113 & $100 \%$ & \\
\hline
\end{tabular}

Notes: Group 1 - patients diagnosed with benign paroxysmal vertigo of childhood; group 2 - patients with diagnosis of vestibular migraine; group 3 - patients with other diagnoses.

by gender is not observed in the literature, ${ }^{2,5,9,10,15}$ but in the present study there was a slight predominance of females.

The predominant diagnosis was of BPVC, found in 49 cases (41.9\%), followed by VM in 16,2\% (19) of the cases. Benign paroxysmal vertigo of childhood is considered an early manifestation of VM, which is recognized by the International Headache Classification (ICHD-2). ${ }^{16}$ Vestibular migraine is diagnosed more frequently in children (35\%) than in adults (6\%), ${ }^{17}$ and, in the present study, its diagnosis followed the 2012 Bárány Society criteria. ${ }^{18}$

Benign paroxysmal vertigo of childhood has been observed in the preschool age group, while VM in most cases presents in schoolchildren, as described by Niemensivu et $\mathrm{al}^{10}$ and Ralli et al. ${ }^{11}$

Benign paroxysmal vertigo of childhood is considered a precursor to migraine, $2,3,9,10,13$ and is clearly distinguished from BPPV, since the latter condition is rare in children; ${ }^{13}$ in the present study, for example, we only found 2 cases of BPPV.

For some authors, such as Martín Sanz and Barona de Guzmán, ${ }^{5}$ migrainous headache is the clinical characteristic that most differentiates groups of pediatric and adult patients.

The prevalence of family history of migraine in the present study was of $52,13 \%$ (61) for all patients, and of $75,51 \%$ (37) in the group with BPVC. Some series show that prevalence in the group of patients with BPVC varies from $55 \%$ to $90 \%{ }^{2,5,10}$

The prevalence of an associated diagnosis of motion sickness was observed mostly in the patients with a diagnosis of BPVC. Martín Sanz and Barona de Guzmán ${ }^{5}$ mentioned motion sickness as a trigger for migraine.

In the present study, we observed a low prevalence of SOM, only $1(1,17 \%)$ case, although some authors have reported SOM as one of the main causes of dizziness in childhood. ${ }^{2,10,15}$ Bower and Cotton ${ }^{15}$ describe SOM as the leading cause of dizziness, followed by BPVC. We attribute the low prevalence found in the present study to the fact that our clinic is specialized, and all the children we examine have already been evaluated by a pediatric otorhinolaryngology clinic, and middle ear diseases have already been ruled out.

The present study did not contain any cases of vestibular neuronitis, which suggests a lower prevalence in the population studied; alternatively, because emergency care is available where our study was performed, these acute cases may have been treated and followed with clinical resolution, and were not referred to the specialized clinic. 
After the diagnosis of BPVC, VM, and motion sickness, the most prevalent diagnosis we found was MVS, in 7 (6\%) patients. This was not found in the literature, but we consider MVS to be one of those conditions suggestive of peripheral vestibular syndrome that are not explained by other causes, and are accompanied by poor eating habits associated with changes in glucose and/or insulinemic curves, and respond to treatment via dietary corrections alone.

As for the clinical condition, 63 (53.84\%) patients complained of vertigo. Most patients (67.34\%) with BPVC complained of vertigo, which is supported by Szirmai. ${ }^{13}$

Headache was reported by 76,06\% (89) of the patients, and among patients with BPVC the prevalence was even higher $87,67 \%$ (43); some authors found lower rates of headache, ${ }^{10,13,15}$ and others reported rates as high as $84 \%{ }^{12}$

Even though the diagnosis of vestibular disorders is known to be clinical, ENG is visibly important in the diagnosis, especially in ruling out central causes and characterizing the type of vestibular disorder found. In the present study, the caloric tests showed alterations in 76,9\% (46) of the cases. Sousa et $\mathrm{al}^{4}$ found altered caloric test results in $86.7 \%$ of their patients, and, in most cases, they also found bilateral vestibular hyperreflexia as a result. Formigoni et $\mathrm{al}^{2}$, performing caloric tests in patients older than 4 years of age and only the rotary decreasing pendular test in younger patients, found alterations in $90 \%$ of the caloric tests, and stated that the rotary test has a sensitivity of $72 \%$. Szirmai ${ }^{13}$ found altered ENG results in $81 \%$ of the cases, and Bower et $\mathrm{a}^{15}$ found alterations in $60 \%$. Most of these authors advocate the routine use of this vestibular examination in children. The cases of BPVC and VM in the present study mostly presented normal vestibular function or bilateral hyperreflexia in the caloric tests. Lanhagem et $\mathrm{al}^{19}$ found altered caloric test results in $21 \%$ of the pediatric patients evaluated with VM; although there are no alterations in vestibular function tests which are characteristic of VM, the finding of hyperreflexia in caloric test can be suggestive. ${ }^{17,20,21}$

Many children were unable to tolerate the caloric tests, showing the importance of conducting exams such as the VHIT in this population. Young ${ }^{1}$ reported low tolerance for caloric tests among children because this examination produces nausea, vomiting, and aspiration hazards, and recommended performing this test in children older than 5 years of age. The VHIT can be applied to children over 2 years of age, is better tolerated, and takes less time to perform. ${ }^{22}$

As for diet, 66.7\% (78) of the patients had poor eating habits, with high carbohydrate intake predominating 45 (57,95\%), followed by prolonged fasting in 21 (23.86\%) cases. We considered inadequate diet to include prolonged fasting with high consumption of rapidly absorbed carbohydrates, like sweets, breads, and pasta, and habitual consumption of foods with high caffeine content. Few studies describe the importance of proper diet in childhood vestibular disorders.

Family history of headache, dizziness, hearing changes, epilepsy or diabetes mellitus was present in 76 (64.95\%) patients, predominantly history of migraine 91 (77,63\%), which is corroborated by Abu-Arafeh and Russell. ${ }^{3}$
In the present study, clinical improvement was observed in $60(51,28 \%)$ of the patients, while rates up to $80 \%$ have been reported by other authors. ${ }^{15}$

Improvement was observed in $50(50.5 \%)$ patients after dietary orientation alone, and only $27(23,23 \%)$ required pharmaceutical treatment combined with dietary changes. The medications used in this series were migraine prophylactics, namely flunarizine, propranolol, and valproic acid. There are few studies with evidence for prophylactic drug treatment for migraine, and none of the drugs are approved by the FDA for pediatric use. ${ }^{23}$ Some studies report benefits of the careful use of propranolol, amitriptyline, nortriptyline, and valproic acid in girls (because these drugs may cause infertility), and flunarizine if the mood and weight of the patient are monitored. ${ }^{24}$ During acute crises, the medications of choice to stop migraine in teens are ibuprofen and triptans. ${ }^{25}$

Another factor to consider in the management and followup of patients with VS is the action of a multidisciplinary team; specifically, cooperation with speech therapy and neurology teams is essential for successful drug treatment and vestibular rehabilitation therapy.

\section{Conclusion}

Most patients evaluated in the present study were schoolage children, with a slight predominance of females; they were diagnosed with BPVC, mostly with dizziness-type VS, family history of migraine, poor eating habits, and normal vestibular function. In most cases, the VS improved after dietary guidance alone, and, in some cases, in association with vestibular rehabilitation therapy.

\section{Conflict of Interests}

The authors have no conflict of interests to declare.

\section{References}

1 Young YH. Assessment of functional development of the otolithic system in growing children: a review. Int J Pediatr Otorhinolaryngol 2015;79(04):435-442

2 Formigoni LG, Medeiros IRT, Santoro PP, Bittar RSM, Bottino MA. Diagnostic approach of vertigo in children. Braz J Otolaryngol 1999;65:78-82

3 Abu-Arafeh I, Russell G. Paroxysmal vertigo as a migraine equivalent in children: a population-based study. Cephalalgia 1995;15 (01):22-25, discussion 4

4 Sousa EC, Siller AL, Tuma VC, Ganança CF, Ganança MM, Caovilla $\mathrm{HH}$. Relação entre dificuldades de leitura e escrita e sintomas e sinais de vestibulopatia periférica em crianças em idade escolar. ORL 2008;26:112-117

5 Martín Sanz E, Barona de Guzmán R. [Benign paroxysmal vertigo of childhood: categorization and comparison with benign positional paroxysmal vertigo in adult]. Acta Otorrinolaringol Esp 2007;58(07):296-301

6 Balatsouras DG, Kaberos A, Assimakopoulos D, Katotomichelakis M, Economou NC, Korres SG. Etiology of vertigo in children. Int J Pediatr Otorhinolaryngol 2007;71(03):487-494

7 Wiener-Vacher SR. Vestibular disorders in children. Int J Audiol 2008;47(09):578-583

8 Franco ES, Caetanaelli EB. Avaliação vestibular en crianças sem queixas auditivas e vestibulares, por meio da Vectoeletronistagmografia computadorizada. Arq Int Otorrinolaringol 2006;10:46-54 
9 Riina N, Ilmari P, Kentala E. Vertigo and imbalance in children: a retrospective study in a Helsinki University otorhinolaryngology clinic. Arch Otolaryngol Head Neck Surg 2005;131(11):996-1000

10 Niemensivu R, Kentala E, Wiener-Vacher S, Pyykkö I. Evaluation of vertiginous children. Eur Arch Otorhinolaryngol 2007;264(10): 1129-1135

11 Ralli G, Atturo F, de Filippis C. Idiopathic benign paroxysmal vertigo in children, a migraine precursor. Int J Pediatr Otorhinolaryngol 2009;73(Suppl 1):S16-S18

12 O'Reilly RC, Morlet T, Nicholas BD, et al. Prevalence of vestibular and balance disorders in children. Otol Neurotol 2010;31(09): 1441-1444

13 Szirmai A. Vestibular disorders in childhood and adolescents. Eur Arch Otorhinolaryngol 2010;267(11):1801-1804

14 Hamilton SS, Zhou G, Brodsky JR. Video head impulse testing (VHIT) in the pediatric population. Int J Pediatr Otorhinolaryngol 2015;79(08):1283-1287

15 Bower CM, Cotton RT. The spectrum of vertigo in children. Arch Otolaryngol Head Neck Surg 1995;121(08):911-915

16 Gladstone JP, Dodick DW. Revised 2004 International Classification of Headache Disorders: new headache types. Can J Neurol Sci 2004;31(03):304-314
17 Furman JM, Marcus DA, Balaban CD. Migrainous vertigo: development of a pathogenetic model and structured diagnostic interview. Curr Opin Neurol 2003;16(01):5-13

18 Lempert T, Olesen J, Furman J, et al. Vestibular migraine: diagnostic criteria. J Vestib Res 2012;22(04):167-172

19 Langhagen T, Lehrer N, Borggraefe I, Heinen F, Jahn K. Vestibular migraine in children and adolescents: clinical findings and laboratory tests. Front Neurol 2015;5:292

20 Cutrer FM, Baloh RW. Migraine-associated dizziness. Headache 1992;32(06):300-304

21 Dieterich M, Brandt T. Episodic vertigo related to migraine (90 cases): vestibular migraine? J Neurol 1999;246(10):883-892

22 Jahn K, Langhagen T, Heinen F. Vertigo and dizziness in children. Curr Opin Neurol 2015;28(01):78-82

23 Kacperski J, Hershey AD. Preventive drugs in childhood and adolescent migraine. Curr Pain Headache Rep 2014;18(06):422

24 Victor S, Ryan S. WITHDRAWN: Drugs for preventing migraine headaches in children. Cochrane Database Syst Rev 2014;11(07): CD002761

25 Richer L, Billinghurst L, Linsdell MA, et al. Drugs for the acute treatment of migraine in children and adolescents. Cochrane Database Syst Rev 2016;4:CD005220 